\title{
Immunocytochemical Localization by Electron Microscopy of 2',3'-Cyclic Nucleotide 3'-Phosphodiesterase in Developing Oligodendrocytes of Normal and Mutant Brain
}

\author{
Peter E. Braun, ${ }^{1, a}$ François Sandillon, ${ }^{2}$ Aled Edwards, ${ }^{1}$ Jean-Marie Matthieu, ${ }^{3}$ and Alain Privat ${ }^{2}$ \\ 'Department of Biochemistry, McGill University, Montreal H3G 1Y6, Canada, ${ }^{2}$ CNRS LP 8402, INSERM U.249, Institute de \\ Biologie, 34060 Montpellier Cedex, France, and 'Laboratoire de Neurochimie, Service de Pédiatrie, Centre Hospitalier \\ Universitaire Vaudois, $\mathrm{CH}-1011$, Lausanne, Switzerland
}

\begin{abstract}
Oligodendrocytes or their putative progenitors were the only cells found to be immunoreactive to polyclonal antisera against the enzyme $2^{\prime}, 3^{\prime}$-cyclic nucleotide $3^{\prime}$-phosphodiesterase (CNP) in developing and mature brains of rats and mice, as visualized by light and electron microscopy. Prior to myelination (day 6), oligodendrocytes of the corpus callosum have reticular networks of CNP-containing filopodia, in addition to abundant CNP throughout the cytoplasm. Some glioblast-like cells of the subventricular zone are also immunoreactive to anti-CNP, suggesting that, as progenitors of oligodendroglia, they express this myelination-related protein as one of the earliest events in myelinogenesis. Following the commencement of myelination (day 15), many oligodendrocytes lose much of their lacelike network of fine projections, possessing, instead, larger CNP-filled processes that extend to myelin-bearing fibers. CNP was always found only in the cytoplasm-containing compartments of the cells and myelin sheaths; neither lamellae nor cellular membranes were immunostained. These data support our contention that CNP is not an intrinsic membrane protein, despite its strong interaction with membrane components when cells are disrupted. In mutant (mld) mice (day 25), the many distended and uncompacted oligodendroglial processes that invest axons with only a few turns of membrane contained cytoplasmic CNP, accounting for the elevated levels of CNP activity previously noted in tissue fractions.
\end{abstract}

The oligodendroglial protein that has been least studied in relationship to myelinogenesis is the enzyme $2^{\prime}, 3^{\prime}$-cyclic nucleotide $3^{\prime}$-phosphodiesterase (CNP; EC 3.1.4.37). This protein, comprising $4-5 \%$ of the total protein of isolated CNS myelin, is the first of the major myelin-related proteins to appear in developing brain (Sprinkle et al., 1978). Although the physio-

\footnotetext{
Received Sept. 22, 1987; revised Nov. 30, 1987; accepted Dec. 3, 1987.

We express our gratitude to all members of our laboratories for their assistance from time to time, especially to N. Rajaofetra for the perfusion of animals. We thank S. Camalon for typing the manuscript and J. R. Teilhac for expert photography. P.E.B. wishes to acknowledge with gratitude his host, Dr. J. Demaille, during his sabbatical leave, and the financial support of INSERM, the Medical Research Council of Canada and the Multiple Sclerosis Society of Canada. P.E.B. was the recipient of a Canada-France Exchange Fellowship.

Correspondence should be addressed to Dr. Braun, McGill University, Department of Biochemistry, 3655 Drummond Street, Montreal H3G 1Y6, Quebec, Canada.

$\therefore$ On sabbatical leave at the laboratory of Dr. A. Privat. Address correspondence to Montreal, as above.

Copyright (C) 1988 Society for Neuroscience $0270-6474 / 88 / 083057-10 \$ 02.00 / 0$
}

logical function of CNP remains unknown, the deposition of this protein in brain parallels the developmental accumulation of myelin and thus has served as a useful biochemical marker for myelin membrane in vitro (Kurihara and Tsukada, 1967; Olafson et al., 1969; Braun and Barchi, 1972).

Despite the fact that $60 \%$ or more of the enzyme activity is present in subcellular fractions of brain that contain myelin, there has been considerable doubt about its actual presence in compact lamellae of the sheath (Shapira et al., 1978; Danks and Matthieu, 1979). Although an earlier investigation (Golds and Braun, 1976) did not clarify the topological relationship of CNP to the myelin bilayer, a recent study (A. Edwards and P. E. Braun, unpublished observations) revealed that this protein is synthesized on free ribosomes, suggesting that it is likely extrinsic to the membrane. Furthermore, immunocytochemical studies at the light-microscope level showed that the external surface of oligodendroglial and Schwann cell plasma membrane does not bind any antibody to CNP, whereas the cytoplasm of these cells immunostains more or less uniformly (McMorris et al., 1984; Yoshino et al., 1985).

It has been reported (Matthieu et al., 1984) that the dysmyelinating mutant "mld" mouse has abnormally high levels of CNP activity associated with isolated brain myelin. This mutation affects the regulation of the gene for myelin basic protein (MBP). It is observed phenotypically in morphologically abnormal oligodendrocytes and myelin, characterized by the virtual absence of MBP during the first 4 weeks, followed by the delayed accumulation of this protein. At a time when normal, compact lamellar myelin ought to appear, in the "mld" brain the oligodendroglial processes engulf axons in a manner that results in only a few, loose, uncompacted wrappings, with abundant inclusions of cytoplasm (Matthieu et al., 1984; Roch et al., 1986). It has not been possible to demonstrate biochemically whether these cytoplasmic compartments in myelin are responsible for this high CNP activity.

To date, the localization of CNP in brain has not been attempted at early developmental stages, prior to the commencement of myelination; on the other hand, immunostaining of myelin sheaths and oligodendrocytes in myelinated brain sections has previously been observed by light microscopy (Nishizawa et al., 1981; Sheedlo and Sprinkle, 1983; Fujishiro et al., 1986), but ultrastructural localization has not been addressed. In view of the growing awareness that this protein is a critical component of myelinogenesis, our objective was to localize CNP by light- and electron-microscopic immunocyto- 
A

Figure 1. Immunoreactive characteristics of antiserum to CNP. Proteins of mouse brain myelin (lane 1), rat brain myelin (lane 2), and purified bovine brain CNP (lane 3) were separated by SDS-PAGE $(12 \%$ gel). Proteins were visualized by Coomassie blue $(A)$ or by immunoreactivity with anti-CNP (1: $1000)$ on a Western blot $(B)$. CNP1 (lower band) and CNP2 (upper band) are indicated by arrowheads.

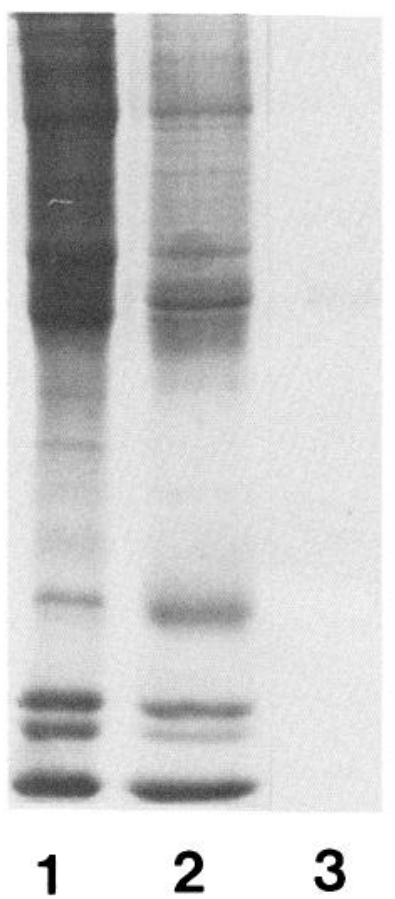

B

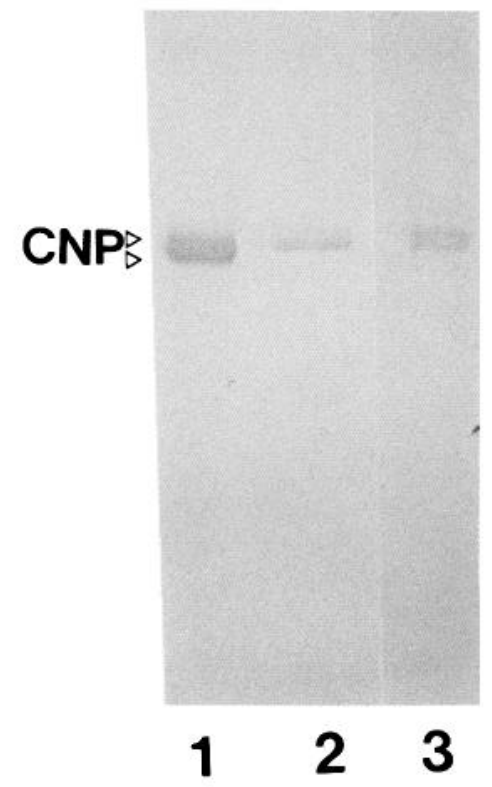

chemistry in (1) normal oligodendroglia or their precursors prior to myelinogenesis and at various stages in myelination, and (2) abnormal oligodendroglia and myelin and dysmyelinating mutant "mld" mice. The results show that CNP is an excellent specific marker for the earliest stages of oligodendroglial development; its detection by antibodies permits the visualization of cellular details not previously seen in vivo and suggests that further study of this protein would contribute to an understanding of early progenitor cell development in relation to the appearance of oligodendrocytes (Privat and Leblond, 1972; Raff et al., 1983; Hirayama et al., 1984; Goldman et al., 1986).

\section{Materials and Methods}

Animals and tissue processing. Sprague-Dawley rats and mice (mld and controls, provided by the laboratory of J. M. Matthieu) were anesthetized with ether and perfused through the heart with heparin and $1 \%$ sodium nitrite, followed by a fixative solution of $4 \%$ paraformaldehyde and $0.2 \%$ glutaraldehyde, in $0.12 \mathrm{M}$ phosphate buffer, $\mathrm{pH} 7.4$. Brains (and in some cases optic nerves and spinal cord) were removed and held in the same fixative at $4^{\circ} \mathrm{C}$ for $1-2 \mathrm{hr}$.

Pre-embedding staining. Sections $(50 \mu \mathrm{m})$ were cut with a Lancer vibrating microtome and processed immunocytochemically for light microscopy with the Vectastain ABC kit or the Auroprobe LM kit with silver enhancement by IntenSE (Janssen Pharmaceutica, Belgium).

Vibratome sections from animals $15 \mathrm{~d}$ and older were pretreated for $1-3 \mathrm{~min}$ in a commercial preparation (Gibco) of trypsin $(0.05 \%$ with $0.02 \%$ EDTA, buffered with Pack's medium) to enhance penetration of the antibody. Sections from younger animals were not pretreated. Briefly, the sections were then incubated sequentially in (1) rinse buffer $(0.06$ M phosphate, $\mathrm{pH} \mathrm{7.6)} \mathrm{twice,} 5$ min each; (2) primary rabbit antiserum diluted in $1 \%$ normal goat serum and phosphate buffer, overnight at $4^{\circ} \mathrm{C}$; (3) rinse buffer $(0.05 \mathrm{~m}$ Tris- $\mathrm{HCl}$ in $0.15 \mathrm{M} \mathrm{NaCl}, \mathrm{pH} 7.6)$ twice for $10 \mathrm{~min}$ each; and (4) reagents of the Vectastain ABC or Auroprobe LM kits, according to the procedures recommended by the manufacturer. For the $\mathrm{ABC}$ procedure, we used $0.05 \%$ of $3,3^{\prime}$-diaminobenzidine in the Tris-sodium chloride buffer (freshly prepared and filtered through paper) with $0.002 \%$ hydrogen peroxide for 3-7 min. For the Auroprobe
LM procedure, the $5 \mathrm{~nm}$ colloidal gold particles were visualized for light microscopy by silver enhancement with IntenSE. Mounted sections were examined with an Olympus Vanox microscope.

For electron microscopy, the immunostained sections were transferred to $1 \% \mathrm{OsO}_{4}$ in $0.12 \mathrm{M}$ phosphate, $\mathrm{pH} 7.2$, and held for $30 \mathrm{~min}$, followed by dehydration in an ascending series of ethanol and then in acetone; they were then embedded in Araldite. Thin sections (50-70 $\mathrm{nm}$ ) were cut on an LKB ultramicrotome and mounted on copper grids. Sections were counterstained with uranyl acetate and lead citrate and examined in a Jeol 2000 EX electron microscope.

Antibodies. Two different rabbit polyclonal antisera were used. One, prepared in our laboratory, was against bovine CNP that had been partially purified by the method of Drummond et al. (1978) and further purified by preparative SDS-PAGE. CNP $(1 \mathrm{mg})$ in complete Freund's adjuvant was injected intramuscularly, followed by 3 subcutaneous injections of $1 \mathrm{mg}$ each in Freund's incomplete adjuvant after 1, 3, and 6 weeks. Rabbits were bled after 7 weeks. The antiserum, at dilutions of $1: 1000$, recognized only CNP on a Western blot of myelin proteins (Fig. 1). We also used antiserum to CNP, generously provided by Dr. F. A. McMorris and David Raible, Wistar Institute, Philadelphia, PA. These antisera provided identical immunocytochemical staining of CNP.

\section{Results}

\section{Immunoreactive CNP in premyelinating rat tissue}

We observed abundant immunostaining of CNP in cells in the corpus callosum at least $5 \mathrm{~d}$ before there was any myelin in this region (Fig. $2 A$; compare with Fig. $2 B$, showing a negative response of the corpus callosum to nonimmune serum). At higher magnification there were numerous immunoreactive oligodendrocytes in various stages of development, often with spectacular arrays of fine filopodia and lamellipodia (Fig. 2C). At this age we also observed occasional pairs of CNP-containing cells with closely apposed cytoplasmic compartments that appeared to be joined-perhaps not yet fully separated postmitoticallyin contrast to cells that are lined up in linear arrays in tissue where myelination has already commenced. Figure $2 D$ shows 

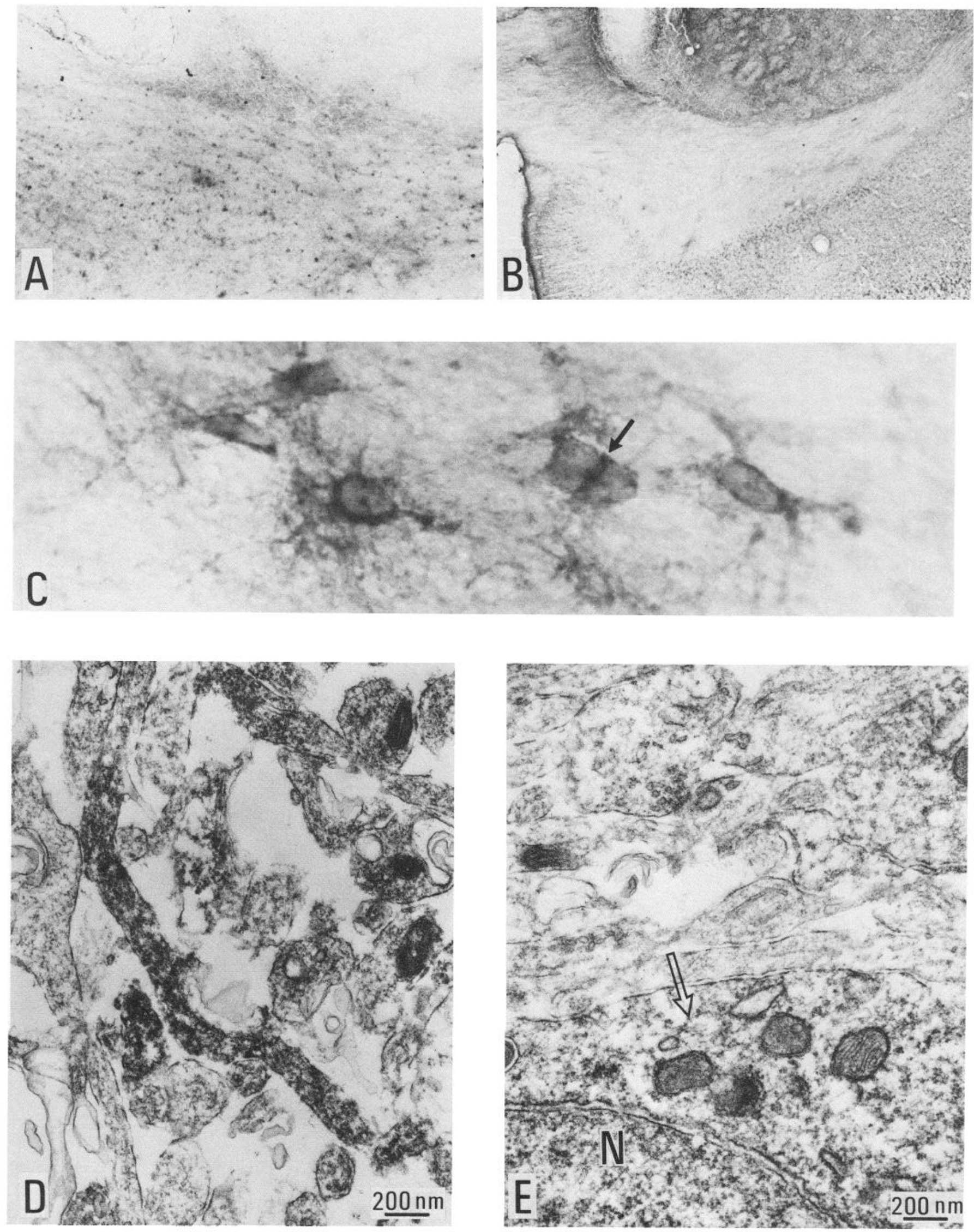

Figure 2. Anti-CNP-reactive cells prior to the onset of myelination in $6 \mathrm{~d}$ rat. $A$, Immunostained cells in the corpus callosum (anti-CNP, 1:2000). Original magnification, $\times 70 . B$, Lack of immunostaining in the corpus callosum by a nonimmune serum $(1: 500)$. Original magnification, $\times 70$. $C$, Immunostaining of immature oligodendrocytes and their filopodia in the corpus callosum. Arrow indicates immunostained, adjoining cytoplasmic compartments of 2 cells (anti-CNP, 1:2000). Original magnification, $\times 1350 . D$, Electron micrograph of oligodendroglial reticular filipodia showing the distribution of immunoreactive CNP (anti-CNP, 1:1000). E, Electron micrograph of a portion of an immature oligodendrocyte, showing part of the nucleus $(N)$ and the cytoplasm (arrow), with its content of immunoreactive CNP (anti-CNP, 1:1000). 

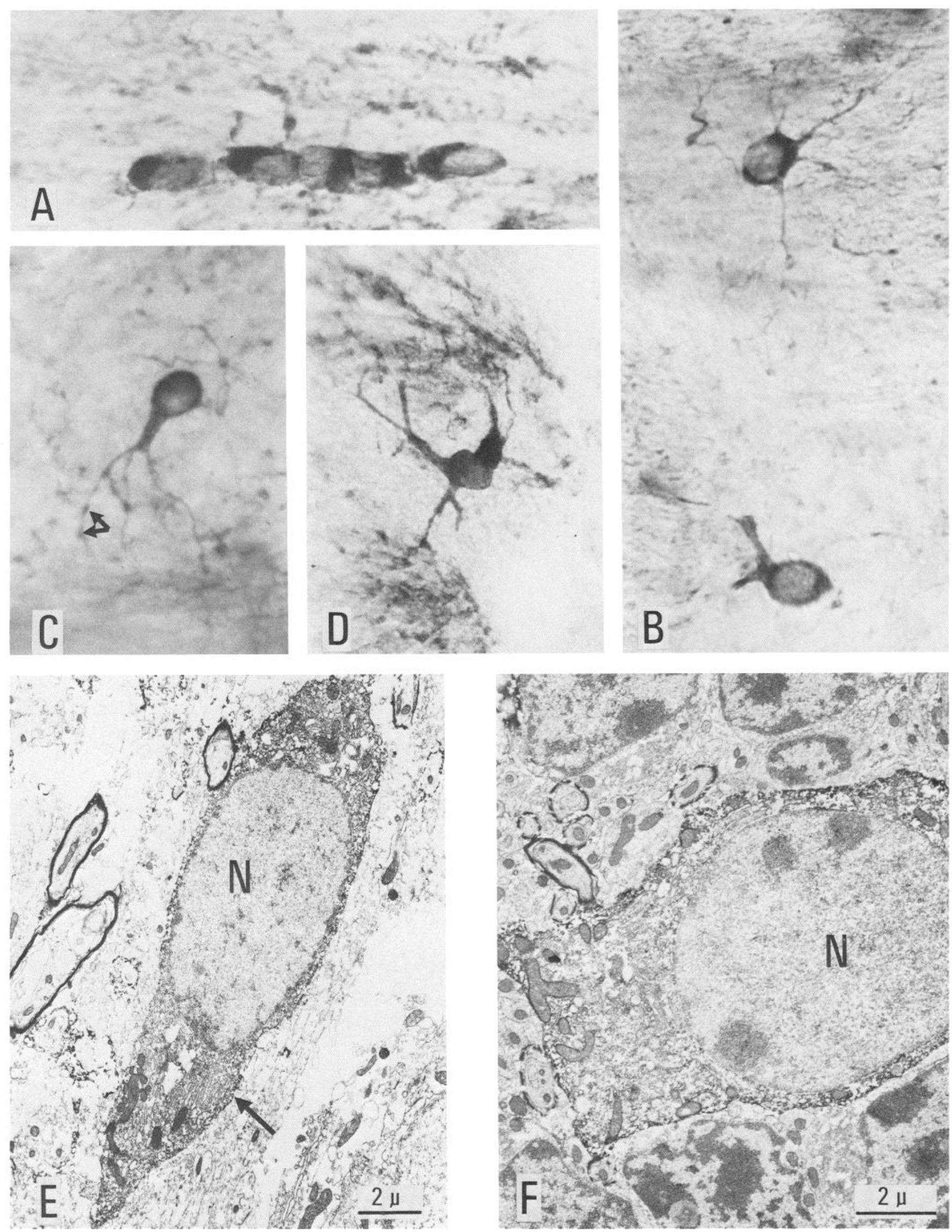

Figure 3. Anti-CNP-reactive oligodendrocytes during active myelination in $15 \mathrm{~d}$ rat brain and in fully myelinated mouse brain. $A$, Immunostained interfascicular oligodendrocytes in the corpus callosum of $75 \mathrm{~d}$ mouse (anti-CNP, 1:1000). Original magnification, $\times 1250$. $B$, Two typical im- 

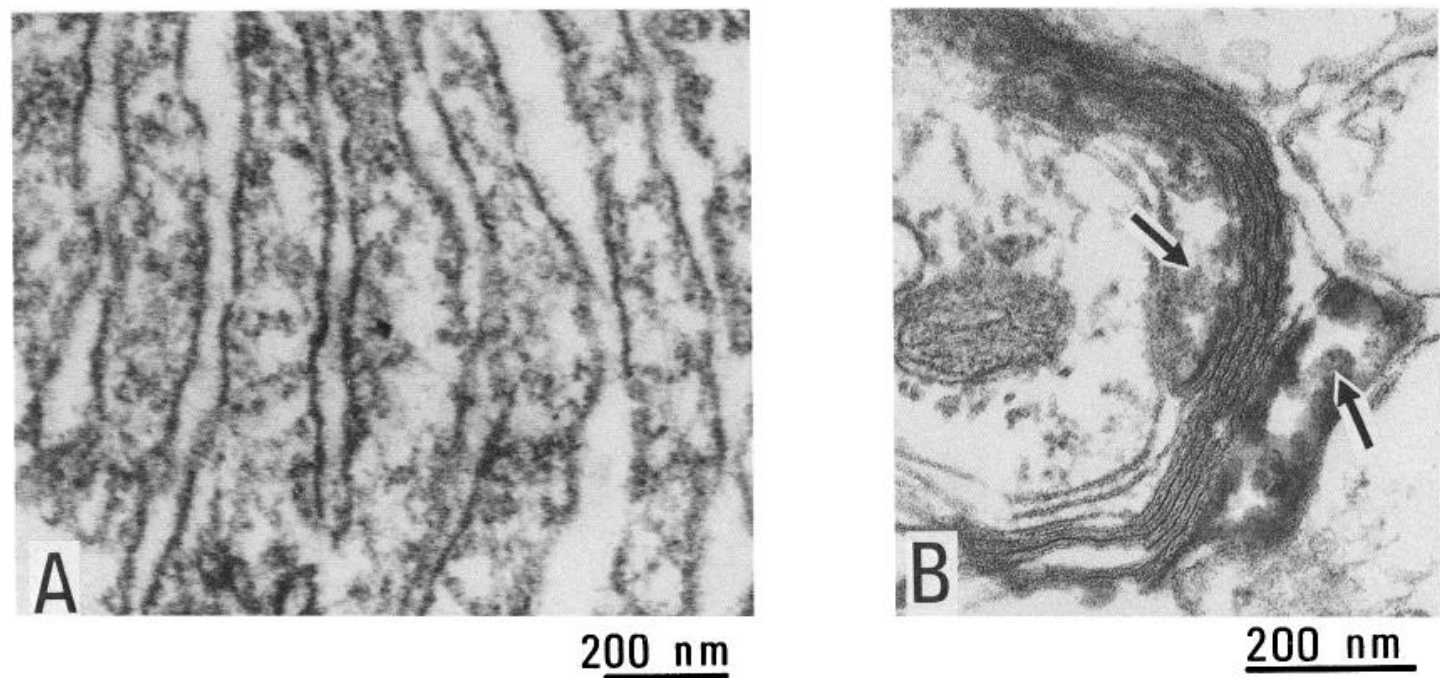

Figure 4. Electron micrographs showing immunoreaction product to CNP in $15 \mathrm{~d}$ rat brain (anti-CNP, 1:1000). A, Endoplasmic reticulum of the immunostained oligodendrocyte seen in Figure $3 E$ (at arrow). Reaction product is present everywhere in the cytoplasm between membranes, but is absent from the lumen. $B$, Cross section of a myelinated axon showing reaction product in the cytoplasm of the inner and outer loops (arrows).

that this elaborate, reticular network can be demonstrated by electron microscopy, which reveals transverse and longitudinally sectioned filopodia from 100 to $300 \mathrm{~nm}$ in diameter, evenly filled with immunoreactive CNP. Similarly, electron micrographs revealed perikarya with characteristics of immature oligodendrocytes, such as a thin cytoplasmic compartment containing many free ribosomes, few isolated cisternae of endoplasmic reticulum, and an abundance of immunoreactive CNP distributed throughout the cytoplasm (Fig. 2E).

\section{Immunoreactive CNP during myelination in the rat}

Figure $3 A$ demonstrates the presence of cytoplasmic CNP in a row of oligodendrocytes in a manner typical of corpus callosum. Although immunostained processes are also visible in adult tissue, they are much more evident at earlier ages, for example, in the prominent oligodendrocytes seen in Figure 3, $B-D$. These well-defined cytoplasmic extensions (some are branched) undoubtedly terminate on axons that are undergoing various stages of myelination (beginning at day 11 in this tissue). In contrast to less mature cells (compare Fig. $2 C$ ), these no longer manifest the intricate, lacelike filopodia seen prior to the envelopment of axons. Figure $3 C$ shows an oligodendrocyte from which several processes are extended to myelinated fiber bundles of the caudate nucleus. At least one of these processes has several discrete distensions (varicosities) of the type previously referred to as gliosomes in cultured oligodendroglia (Szuchet and Dumas, 1983). A new procedure for visualizing immune complexes in tissue by silver intensification of a colloidal gold-labeled second antibody circumvents some of the disadvantages of peroxidasegenerated aggregates of reaction product, and, in Figure $3 D$, provides evidence supportive of the distribution of CNP in oligodendroglial processes extending to caudate fibers.

Electron microscopy confirms the presence of immunoreactive CNP throughout the cytoplasm at 2 different ages, in 2 tissues (Fig. 3, E,F), with a distinct concentration at the periphery, generally adjacent to the plasma membrane. At a higher magnification of immunolabeled endoplasmic reticulum (Fig. $4 A$ ), we noted the absence of CNP from the lumen, with a generally random dispersion in the cytoplasmic spaces. In wellmyelinated fibers, CNP was evident in the inner and outer membrane loops ("tongues") of the sheath (Fig. $4 B$ ), but the lack of sufficiently large, accessible spaces between compact lamellae precluded a direct assessment of the protein in this region.

\section{Immunoreactive CNP in the mld mouse}

With the delayed commencement of myelination in the corpus callosum at day 25 in the mld mutant mouse, the initial engulfment of axons by the cytoplasmic extensions of oligodendrocytes can be visualized by immunostaining for CNP (Fig. $5 A$ ). Individual large cells with their processes are easily recognized (especially at higher magnification) at the margins of the corpus callosum, and even in adjacent gray matter regions, but typical, compact myelin has not yet appeared. The intense, diffuse staining is due to the myriad filopodia and processes of oligodendrocytes at the onset of myelination. At day 60 (Fig. $5 B$ ) in these animals, when myelination is in progress, the immunostaining becomes less diffuse, with well-defined cells recognizable throughout the white matter. Since there are fewer filopodia and less cytoplasm associated with compact myelin, the overall effect is a diminished intensity of immunostaining

munostained cells in the corpus callosum of $15 \mathrm{~d}$ rat (anti-CNP, 1:2000). Original magnification, $\times 1625$. C, Immunostained oligodendrocyte in the caudate of $15 \mathrm{~d}$ rat brain. Arrows denote varicosities along prominent processes that terminate on myelinated fibers (anti-CNP, 1:2000). Original magnification, $\times 1625$. $D$, Oligodendrocyte in the caudate of $15 \mathrm{~d}$ rat, immunostained for CNP by the immunogold and silver-intensification procedure (see Materials and Methods) (anti-CNP, 1:2000). Original magnification, $\times 850$. E, Electron micrograph of an immunostained oligodendrocyte in the corpus callosum of $15 \mathrm{~d}$ rat. Arrow indicates region of the perikaryon that contains abundant, well-preserved endoplasmic reticulum (compare Fig. 4A). $N$, nucleus (anti-CNP, 1:1000). $F$, Electron micrograph of an oligodendrocyte in the granular layer of the cerebellum of $15 \mathrm{~d}$ rat. Immunostaining for CNP is present throughout the perikaryon, with relative concentration of reaction product at the periphery. $N$, nucleus (anti-CNP, 1:1000). 

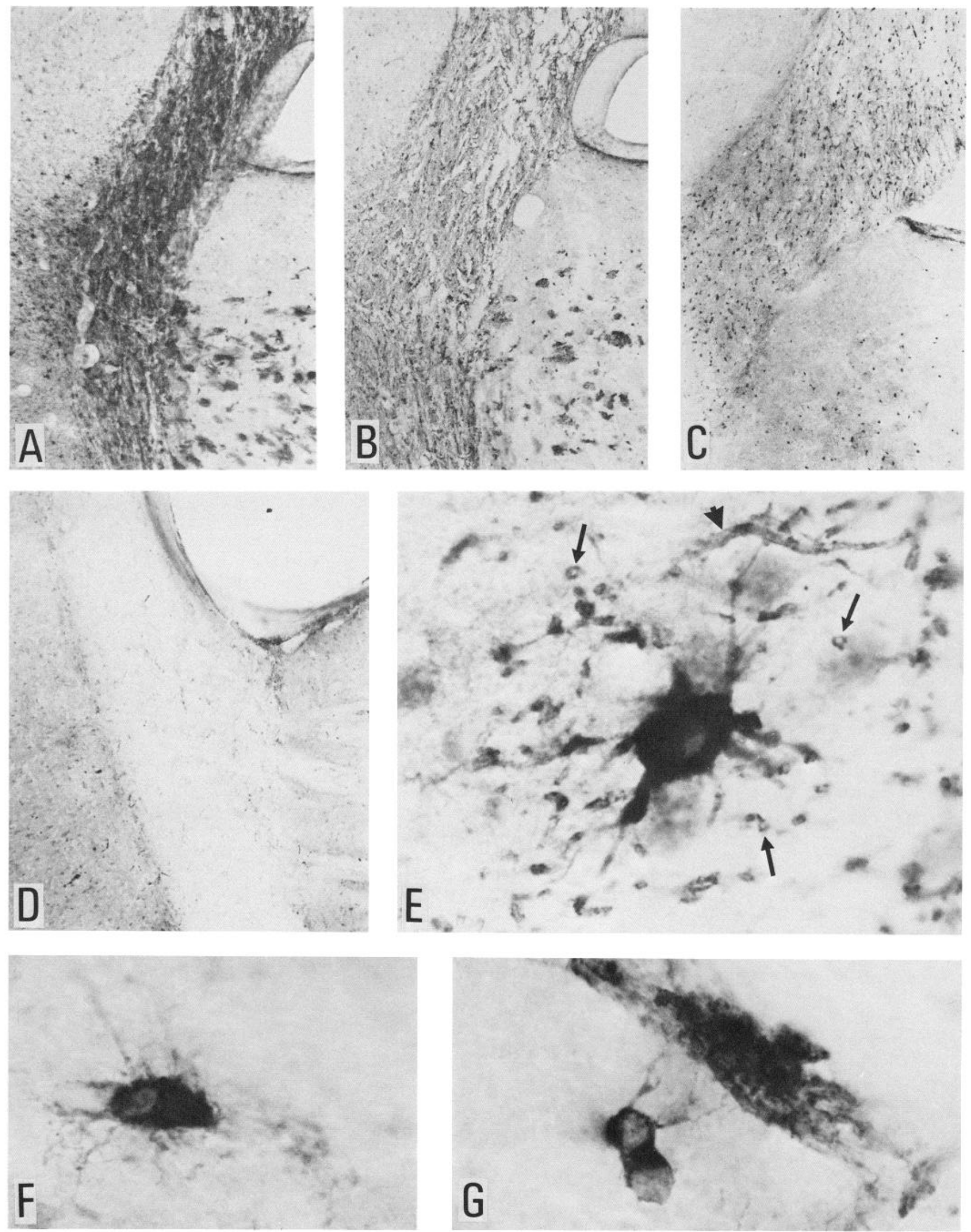

Figure 5. Anti-CNP-reactive cells in brains of mutant (mld) mice. $A$, Immunostaining of the corpus callosum at $25 \mathrm{~d}$ (anti-CNP, 1:2000). Original magnification, $\times 65$. B, Same as $A$, at $60 \mathrm{~d}$. $C$, Immunostaining of the corpus callosum at $75 \mathrm{~d}$ in a normal mouse. Original magnification, $\times 65$. 
for CNP. Comparison with the fully myelinated corpus callosum of a normal, adult mouse (Fig. $5 \mathrm{C}$ ) shows that, in the latter, immunostaining is even more discrete because CNP is accessible essentially only in the oligodendroglial perikarya. A comparable control section exposed to nonimmune serum shows no immunostaining of white matter tracts (Fig. $5 D$ ).

At higher magnification (Fig. $5 E$ ), a typical oligodendrocyte with short, thick processes and some finer extensions is surrounded by numerous axons in cross section, enveloped by partial or complete rings of CNP immunoreaction product, a consequence of the abnormally large amount of cytoplasm in the processes that engulf the axons. In addition, at this age we also observe (Fig. $5 F$ ) numerous young oligodendrocytes with complex networks of filopodia but without the thicker processes of more mature cells (compare with Figs. $2 C$ and $3, B, C$ ). At day 60 (Fig. $5 G$ ), the mass of cytoplasmic projections is greatly diminished, with myelinating processes in evidence, confirming the reason for the reduced staining of CNP in Figure $5 B$.

The relative concentrations of CNP at the margins of the cell are again evident in electron micrographs of a white matter oligodendrocyte (Fig. 6A) and a gray matter "satellite" cell (Fig. $6 B$ ), resembling the distribution in cells of normal animals.

Of particular interest to us was the presence of CNP in the small cytoplasmic compartment of some subventricular (subependymal) cells (Fig. 6C) that did not yet have any other charactcristics of oligodendrocytes. The extensive immunostaining of CNP in the cytoplasmic processes that engulf axons (Fig. 5E) is verified by Figure $6 D$, where immune complexes of this protein are seen among 3 turns of membrane around an axon.

\section{Discussion}

The corpus callosum in the rat at days 6 and 7 was selected for its abundance of oligodendrocytes at all stages of early development, well before the first appearance of myelin (day 11; Sturrock, 1980). At this age we observed many immunostained oligodendrocytes, often bearing remarkably intricate, fine processes or lacelike filopodia reminiscent of cells grown in culture (Collins and Seeds, 1986; Kachar et al., 1986; Knapp et al., 1987). This is significant because the question has been raised as to whether or not the intricate network of fingerlike projections observed in vitro is actually present in vivo.

The retraction of these lacy networks, coincident with oligodendrocyte maturation, when processes engage and myelinate axons, has also been noted by Friedrich (1986), who has speculated that the array of fine projections could represent a mechanism by which oligodendrocytes sample their environment and locate axons to be myelinated.

Oligodendroblasts that contained CNP immunoreaction product were also occasionally observed by both light and electron microscopy, especially in the subventricular layer. These round cells, with their sparse cytoplasm and without the prominent filopodia or processes of more mature cells, might otherwise have been simply designated as glioblasts. In some sections of this region, there were clusters of these cells with similar morphology, but with only one or 2 of them immunostained.
Presumably they all had access to the antibody, but, in all likelihood, only those cells programmed to become oligodendrocytes contained CNP. This supports the contention of Privat and Leblond (1972) that differentiation of progenitors may have. already begun in the subventricular zone prior to migration into other regions of the brain. Similarly, we also observed postmitotic "sister" oligodendroblasts, not yet fully separated from each other and just beginning to elaborate a few fine processes; each contained CNP in the thin cytoplasmic compartment surrounding the nucleus.

At more advanced developmental stages, immunostaining by anti-CNP of oligodenroglia and portions of myelin sheaths in white matter regions of brain and in cultures has been previously noted by light microscopy, although not in detail (Nishizawa et al., 1981; Sheedlo and Sprinkle, 1983; McMorris et al., 1984; Fujishiro et al., 1986). In addition to extending these findings in $15 \mathrm{~d}$ rat brain, we have observed the immunostaining of oligodendrocytes (the so-called "satellite" cells) and their processes in gray matter; this attests to the close resemblance of these cells to their myelinating counterparts in white matter and suggests that immunostaining with anti-CNP is an excellent means of visualizing these cells for further investigation.

The relatively broad and diffuse distribution of the peroxidase-generated reaction product throughout the cytoplasm at all developmental stages suggests to us not only a wide distribution of sites of CNP synthesis, but also that CNP has a functional role in the cytoplasm. Since it is present in immature oligodendrocytes before the major myelin proteins, such as MBP and PLP, make their appearance, and remains in abundance in fully mature cells of adult animals, we believe its function could be related not only (indeed, if at all) to the structure of myelin, but even more likely to the process by which myelin is assembled. Our electron micrographs also show this broad distribution of CNP in perikarya and processes, with a relatively greater abundance of CNP in the cell periphery adjacent to, but not superimposed on, the plasma membrane. Furthermore, electron micrographs reveal that this protein is not evidently associated with any intracellular membranes, although we cannot rule out the possibility that juxtaposed reaction product represents protein bound to membrane. By comparison, other myelin proteins, such as MBP, PLP, the myelin-associated glycoprotein, and the cnzyme UDP-gal: ccramide galactosyl transferasc, are distributed differently in the perikaryon and processes of oligodendrocytes (Roussel and Nussbaum, 1981; Nussbaum and Roussel, 1983; Dubois-Dalcq et al., 1986; Roussel et al., 1987). Our finding that the corpus callosum of "mld" mice immunostains to a much greater extent than that of age-matched normal animals supports the biochemical demonstration of a greater than 2-fold elevation in the specific activity of membrane-associated CNP in mld than in normal brain, and shows that this is due to an increased content of this protein in the cytoplasm. Furthermore, the decline in immunoreactivity by day 60 in mld also follows the downward trend in the decline of CNP activity as myelination progresses in this mutant (Matthieu et al., 1984). Additionally, the numerous partial or complete "rings" of im- 

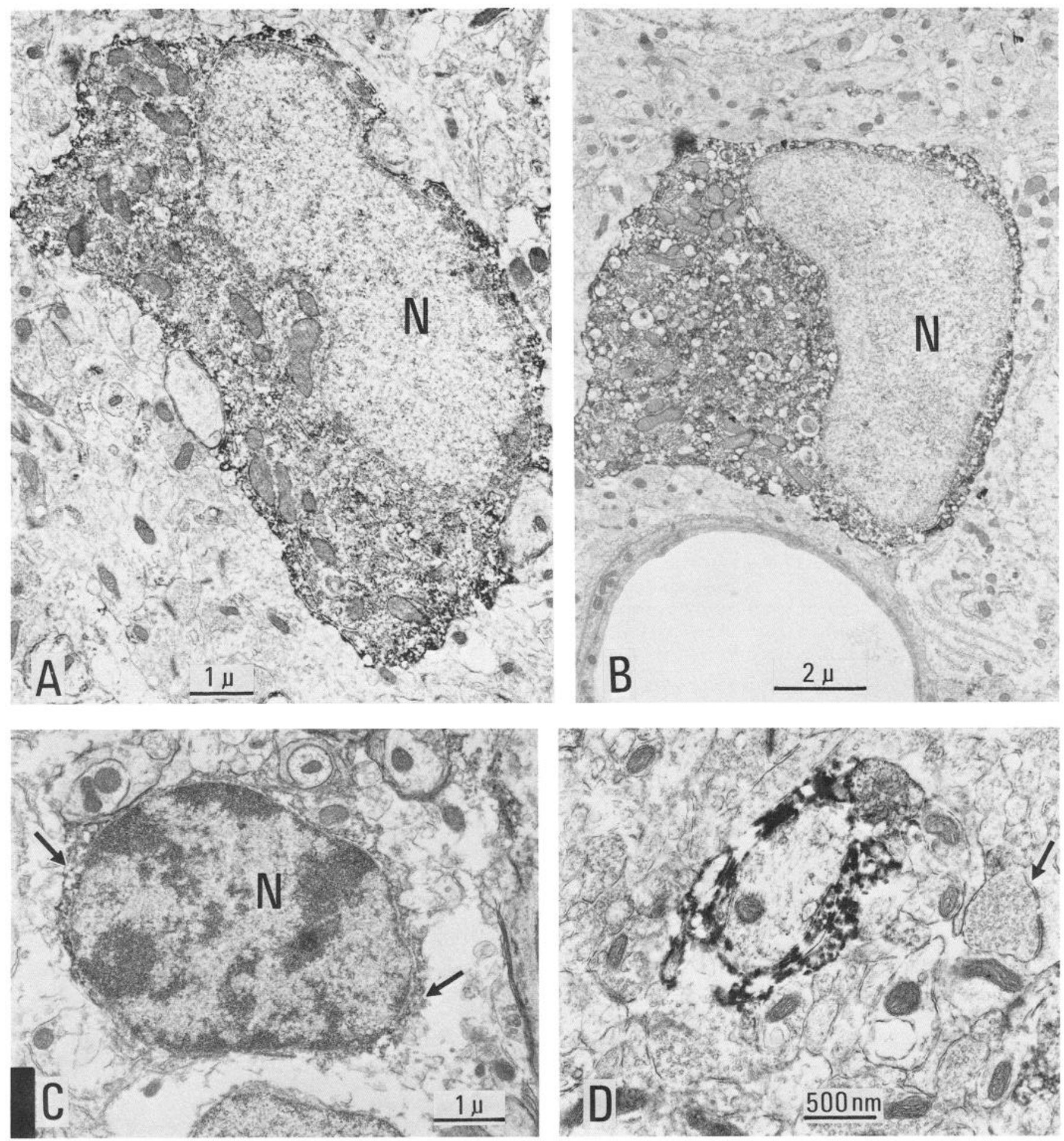

Figure 6. Electron micrographs of cells immunostained for CNP in brains of mutant (mld) mice. $A$, Oligodendrocyte in corpus callosum at $60 \mathrm{~d}$ (anti-CNP, 1:1000). $N$, nucleus. B, Oligodendrocyte adjacent to a blood vessel (below) and a neuron (to the right) in the gray matter at $60 \mathrm{~d}$ (antiCNP, 1:1000). C, Progenitor cell in the subventricular layer at $60 \mathrm{~d}$. Arrows indicate the thin cytoplasmic compartment filled with reaction product (anti-CNP, 1:1000). D, CNP immunoreaction product in the cytoplasm of an oligodendroglial process that has enveloped an axon, in the corpus callosum at $25 \mathrm{~d}$. Arrow indicates the intact membrane and vesicles at an adjacent nerve ending as an indicator of general morphological preservation in this section (anti-CNP, 1:1000).

munoreaction product around axons that are visible at day 20 are diminished at day 60 and nearly absent from well-myelinated controls, a further demonstration that the CNP-containing oligodendroglial extensions that envelop axons and invest them with a few turns of cytoplasm-filled membrane are an early phenomenon in myelination that is exaggerated in this mutant. Our ultrastructural analysis confirmed that the immunoreaction product is actually in the cytoplasmic inclusions and not especially associated with the membrane. This is a significant observation because subcellular fractionation of the brain, 
whether normal or mld, results in a virtually complete association of CNP with isolated membrane. This lends support to our contention that CNP is not an intrinsic membrane protein, but an extrinsic component; that it is rather more like a constituent of a membrane skeleton, reminiscent of the spectrinbased lattice underlying the erythrocyte membrane; or the lamin-based nuclear lamina that lines the inner nuclear membrane (Aebi et al., 1986); or the spectrin network associated with various membranes of mammalian brain (Zagon et al., 1986). Although we never observed CNP immunostaining in lamellae, even when these were relatively uncompacted, we cannot exclude the possibility that CNP is part of the membrane skeleton in the major period region of lamellar myelin, but is not accessible to antibodies. On the other hand, the presence of CNP in the cytoplasm-containing compartments of the sheath accords with a variety of other biochemical observations (Shapira et al., 1978; Danks and Matthieu, 1979) that point to a nonlamellar localization of CNP in the sheath.

Previously published studies on the localization of a myelin protein class designated W1 (Roussel et al., 1978; Roussel and Nussbaum, 1981) are worth noting. The reported immunostaining with the antiserum is, in some figures, reminiscent of our own, but differences are noted as well. Although the anti-W1 cannot be assumed to be identical to anti-CNP, some of the reported immunostaining could be due to anti-CNP contained in the serum. The same reservations about the identity of $\mathrm{W} 1$ apply to the recent studies of the developmental expression of myelin proteins, including W1 (detected by the same anti-W1), in oligodendroglia and myelin (Monge et al,, 1986).

During the past few years, there has been considerable interest in establishing the origins of oligodendrocytes, with an emphasis on in vitro cultures of dissociated brain cells. These have provided interesting and valuable data on progenitor cells and their differentiation into oligodendrocytes or astrocytes and on early development (Barbarese et al., 1983; Raffet al., 1983; Hirayama et al., 1984; Collins and Seeds, 1986; Goldman et al., 1986). Unlike anti-galactocerebroside, anti-CNP can be used for both light- and electron-microscopic studies as an integrative tool with which to evaluate in vivo the validity of interpretations derived from in vitro studies.

\section{References}

Aebi, U., J. Cohn, L. Buhle, and L. Gerace (1986) The nuclear lamina is a meshwork of intermediate-type filaments. Nature $323: 560-564$.

Barbarese, E., S. E. Pfeiffer, and J. H. Carson (1983) Progenitors of oligodendrocytes: Limiting dilution analysis in fetal rat brain culture. Dev. Biol. 96: 84-88.

Braun, P. E., and R. L. Barchi (1972) 2',3'-Cyclic nucleotide 3'-phosphodiesterase in the nervous system. Electrophoretic properties and developmental studies. Brain Res. 40: 437-444.

Collins, M., and N. W. Seeds (1986) Oligodendroglia development in cell culture as monitored with monoclonal antibody. J. Neurosci. 6: 2635-2643.

Danks, D. M., and J.-M. Matthieu (1979) Hypotheses regarding myelination derived from comparisons of myelin subfractions. Life Sci. 24: $1425-1440$.

Drummond, R. J., E. B. Hamill, and A. Guha (1978) Purification and comparison of the $2^{\prime}, 3^{\prime}$-cyclic nucleotide $3^{\prime}$-phosphodiesterases from bovine brain and spinal cord. J. Neurochem. 31: 871-878.

Dubois-Dalcq, M., T. Behar, L. Hudson, and R. A. Lazzarini (1986) Emergence of three myelin proteins in oligodendrocytes cultured without neurons. J. Cell Biol. 102: 384-392.

Friedrich, V. L. (1986) Oligodendrocytes before myelination: Intercellular relationships. Trans. Am. Soc. Neurochem. 17: 255.
Fujishiro, M., S. Kohsaka, N. Kazuhiro, and Y. Tsukada (1986) Production of monoclonal antibody to $2^{\prime}, 3^{\prime}$-cyclic nucleotide 3 '-phosphodiesterase from bovine cerebral white matter. J. Neurochem. 47: 191-195.

Goldman, J. E., S. S. Geier, and M. Hirano (1986) Differentiation of astrocytes and oligodendrocytes from germinal matrix cells in primary culture. J. Neurosci. 6: 52-60.

Golds, E. E., and P. E. Braun (1976) Organization of membrane proteins in the intact myelin sheath. J. Biol. Chem. 251: 4729-4735.

Hirayama, M., P. A. Eccleston, and D. H. Silberberg (1984) The mitotic history and radiosensitivity of developing oligodendrocytes in vitro. Dev. Biol. 104: 413-420.

Kachar, B., T. Behar, and M. Dubois-Dalcq (1986) Cell shape and motility of oligodendrocytes cultured without neurons. Cell Tissue Res. 244: 27-38.

Knapp, P. E., W. P. Bartlett, and R. P. Skoff (1987) Cultured oligodendrocytes mimic in vivo phenotypic characteristics: Ccll shapc, expression of myelin-specific antigens, and membrane production. Dev. Biol. 120: 356-365.

Kurihara, T., and Y. Tsukada (1967) The regional and subcellular distribution of $2^{\prime}, 3^{\prime}$-cyclic nucleotide $3^{\prime}$-phosphohydrolase in the central nervous system. J. Neurochem. 14: 1167-1174.

Matthieu, J.-M., F. X. Omlin, H. Ginalski-Winkelmann, and B. J. Cooper (1984) Myelination in the CNS of mld mutant mice: Comparison between composition and structure. Dev. Brain Res. 13: 149-158.

McMorris, F. A., S. U. Kim, and T. J. Sprinkle (1984) Intracellular localization of $2^{\prime}, 3^{\prime}$-cyclic nucleotide 3 '-phosphohydrolase in rat oligodendrocytes and $\mathrm{C}_{6}$ glioma cells, and effect of cell maturation and enzyme induction on localization. Brain Res. 292: 123-131.

Monge, M., D. Kadiiski, C. M. Jacque, and B. Zalc (1986) Oligodendroglial expression and deposition of four major myclin constituents in the myelin sheath during development. Dev. Neurosci. 8: 222235.

Nishizawa, Y., T. Kurihara, and Y. Takahashi (1981) Immunohistochemical localization of $2^{\prime}, 3^{\prime}$-cyclic nucleotide $3^{\prime}$-phosphodiesterase in the central nervous system. Brain Res. 212: 219-222.

Nussbaum, J. L., and G. Roussel (1983) Immunocytochemical demonstration of the transport of myelin proteolipids through the Golgi apparatus. Cell Tissue Res. 234: 547-559.

Olafson, R. W., G. I. Drummond, and J. F. Lee (1969) Studies on 2',3'-cyclic nucleotide 3'-phosphohydrolase from brain. Can. J. Biochem. 47: 961-966.

Privat, A., and C. P. Leblond (1972) The subependymal layer and neighboring region in the brain of the young rat. J. Comp. Neurol. 146: 277-302.

Raff, M. C., R. H. Miller, and M. Noble (1983) A glial progenitor cell that develops in vitro into an astrocyte or an oligodendrocyte depending on culture medium. Nature 303: 390-396.

Roch, J.-M., M. Brown-Luedi, B. J. Cooper, and J.-M. Matthieu (1986) Mice heterozygous for the mld mutation have intermediate levels of myelin basic protein mRNA and its translation products. Mol. Brain Res. 1: 137-144.

Roussel, G., and J. L. Nussbaum (1981) Comparative localization study of Wolfgram W1 and myelin basic proteins in the rat brain during ontogenesis. Histochem. J. 13: 1029-1047.

Roussel, G., J.-P. Delaunoy, P. Mandel, and J. L. Nussbaum (1978) Ultrastructural localization study of two Wolfgram proteins in rat brain tissue. J. Neurocytol. 7: 155-163.

Roussel, G., J. L. Nussbaum, A. Espinosa de los Monteros, and N. M. Neskovic (1987) Immunocytochemical localization of UDP-galactose: Ceramide galactosyltransferase in myelin and oligodendroglial cells of rat brain. J. Neurocytol. 16: 85-92.

Shapira, R., W. C. Mobley, S. B. Thiele, M. R. Wilhelm, A. Wallace, and R. F. Kibler (1978) Localization of 2',3'-cyclic nucleotide 3'phosphohydrolase of rabbit brain by sedimentation in a continuous sucrose gradient. J. Neurochem. 30: 735-744.

Sheedlo, H. J., and T. J. Sprinkle (1983) The localization of 2': $3^{\prime}-$ cyclic nucleotide $3^{\prime}$-phosphodiesterase in bovine cerebrum by immunofluorescence. Brain Res. 288: 330-333.

Sprinkle, T. J., M. E. Zaruba, and G. M. McKhann (1978) Activity of $2^{\prime}, 3^{\prime}$-cyclic-nucleotide $3^{\prime}$-phosphodiesterase in regions of rat brain during development: Quantitative relationship to myelin basic protein. J. Neurochem. 30: 309-314.

Sturrock, R. R. (1980) Myelination of the mouse corpus callosum. Neuropathol. Appl. Neurobiol. 6: 415-420. 
Szuchet, S., and M. Dumas (1983) An in vitro approach to the study of oligodendrocytes and their involvement in multiple sclerosis. In Multiple Sclerosis, J. Antel, ed., pp. 729-755, Neurology Clinics of North America, Saunders, New York.

Yoshino, J., M. P. Dinneen, T. J. Sprinkle, and G. H. DeVries (1985)
Localization of $2^{\prime}, 3^{\prime}$-cyclic nucleotide $3^{\prime}$-phosphodiesterase on cultured Schwann cells. Brain Res. 325: 199-203.

Zagon, I. S., R. Higbee, B. M. Riederer, and S. R. Goodman (1986) Spectrin subtypes in mammalian brain: An immunoelectron microscopic study. J. Neurosci. 6: 2977-2986. 